\title{
The Hypothesis of Unexplained Brain Damage and Learning Difficulties
}

Theofilidis Antonis

Occupational Therapy Department University of Western Makedonia - Greece

Corresponding Author: Theofilidis Antonis, Occupational Therapy Department University of Western Makedonia - Greece

Received date: February 26, 2021; Accepted date: April 30, 2021; Published date: May 05, 2021

Citation: Theofilidis Antonis (2021) The Hypothesis of Unexplained Brain Damage and Learning Difficulties J. Neuroscience and Neurological Surgery. 8(5); DOI:10.31579/2578-8868/165

Copyright: (C) 2021 Theofilidis Antonis, This is an open-access article distributed under the terms of The Creative Commons Attribution License, which permits unrestricted use, distribution, and reproduction in any medium, provided the original author and source are credited

Abstract:
The term learning disabilities refers to a complex and multidimensional phenomenon that affects many thousands of
students. Due to the rich symptomatology of learning difficulties and the increased differences between individuals, it
has not been possible to analyze all cases exclusively from the perspective of the neuropsychological approach.
Aim. The aim of this study was to present research conducted in the context of theories on the brain function of people
with learning disabilities. Supporting the hypothesis of brain dysfunction.
Methodology: Literature review was carried out in the web, which referred to researches on Special Learning
Disabilities and the brain function associated with them.
Results: Review of the literature highlighted key points of the relationship between learning difficulties and brain
function. Brain dysfunction and the cognitive functions produced emerged as one of the key factors involved in learning
disabilities. Many of the theories developed around the problems of children with learning disabilities have focused on
specific areas of the brain that may be dysfunctional.
Conclusions: The difficulty of locating obvious brain damage in individuals who have been characterized as dyslexic
leads to the strengthening of the hypothesis of the existence of a slight or minimal brain damage that cannot be easily
diagnosed and strengthens the hypothesis of an unexplained brain damage that could be heterogeneous groups of
learning disabilities.
Keywords: learning difficulties; brain; undetectable brain damage

\section{The causes of special developmental learning}

\section{difficulties}

The causes of Learning Disabilities have been sought after by several research and scientific areas while dozens of researches and suggestions have been made on how a learning disability occurs and what it consists of. Many causes have been suggested, reflecting the varied characteristics of students with learning disabilities. Just as there are different types of learning disabilities, so there are likely to be different causes (Renteria, 2012). It is known that the term "Learning Disabilities" has been used for over thirty years in the field of scientific research. It was originally proposed by Sammuel Kirk (1962) to describe the low school performance of children with a normal IQ. Learning disabilities affect a group of disorders affecting language, perception, speech, writing, arithmetic, learning and memory, movement, attention, thinking, reasoning and resolution mechanisms (Renteria, 2012).
These disorders are attributed to dysfunction of the Central Nervous System. The complexity of the Central Nervous System is demonstrated by the billions of nerve cells that make it up and support its function. The way they work is due to the normal or abnormal functioning of the Central Nervous System which, among other things, has an impact on the processing of learning. Well-known theories refer to disorders in certain areas of the Central Nervous System or even to a delay in the maturation of specific nerve cells (Park, \& Lombardino, 2013). Most of the modern researchers agree on the existence of a neurological basis of learning disabilities, recognizing it as the most dominant tendency for their justification. They assume there is an electrochemical transmission of the learning processes in the brain and that it is possible that abnormalities in the electrical waves of the cerebral cortex contribute to the dysfunction of learning processes. When there is no evidence of brain damage (which is true of most children with learning disabilities), the term minimal brain dysfunction is sometimes used, especially by doctors. This wording implies brain damage, arguing that the child's brain is not functioning 
properly (Baillieux, et. al., 2008). Due to the rich symptomatology of learning difficulties and the increased differences between individuals, it has not been possible to analyze all cases exclusively from the perspective of the neuropsychological approach. Thus, in addition to neurobiological factors, there are three other known categories of factors: biochemical, genetic, and environmental. The biochemical factors that are being investigated for their contribution to the genesis of reading disorders and learning disorders in general, argue that biochemical disorders in a child's body cause these difficulties. For example, artificial colors and flavors in many foods that children consume (poor nutrition), eating conditions, the inability of the child's blood flow to synthesize a normal amount of vitamins, etc. Today, most professionals, of course, do not trust the hypothesis of biochemical imbalance as a major cause or treatment of learning disabilities (Park, \& Lombardino, 2013). Genetic factors refer to heredity and the hypothesis that siblings and children of people with reading difficulties have a slightly higher than normal chance of developing reading problems. Although some research has identified a possible chromosomal region for the genetic transmission of phonological deficits that may predispose a child to future reading problems, different conclusions between genetic studies leave the field unclear at this time. As for the environmental factors, they refer to the living of children in conditions of severe deprivation, early in the life of the child, to the limited exposure to highly effective teaching in school, and even to the quality of teaching. Other culprits that have been identified include an unwanted pregnancy, the father's alcoholism, an illegitimate childbearing, and divorce, which can cause learning difficulties (Baillieux, et. Al., 2008). However, although a large body of data shows that the learning problems of many students can be remedied with intensive and systematic teaching, it would be naive to think that learning disabilities are caused solely by inadequate teaching or psychological neglect (Park, \& Lombardino, 2013). .

\section{The one-factor and multi-factor approach}

The theories that are valid today for learning disabilities, accept the neurological basis of special developmental learning disabilities and move around two axes, which at the same time classify them into two general categories. There are theoretical positions with which one tries to justify the learning difficulties in one dimension, ie they emphasize one main factor, and there are positions that use a multidimensional causal approach, ie they emphasize many factors. The one-factor approach, otherwise known as the neurological-biological perspective or medical model of interpreting the phenomenon that is basically one-factor, explores how the brain processes information and, consequently, the points at which children with learning disabilities differ from the whole to the learning processes (eg structure and function of the brain, etc.). It focuses, therefore, on specific areas or parts of the brain that are intertwined with the individual's language development (Renterı, 2012). Proponents of the neuro-biological perspective focus their scientific interest on the anatomy and function of the human brain, that is, the way the brain processes sensory information. However, there are disagreements about how functional impairment manifests itself and what are the functional processes that negatively affect the ability to read and write (Baillieux, et. Al., 2008). The existence of a neurological basis is a commonly accepted hypothesis and many studies have been conducted to determine the exact type of neurological disorder. Some scientists claim that dyslexia is due to brain damage, which is either inherited (developmental dyslexia) or caused by illness or injury (acquired dyslexia). People with developmental dyslexia show either underdevelopment of specific areas of the brain, problems in its general organization, or insufficient differentiation of the functions of the cerebral hemispheres. Also, many children experience insufficient dominance of the left cerebral hemisphere which contributes to the creative use of language (Lagarde, et. al., 2009). Causes of learning disabilities include minimal neurological dysfunction, slowing of neurological maturation of the brain, ectopic growth of neurons in the cerebral cortex, inhibition of left hemisphere growth, and compensatory development of right cerebral hemispheres, cerebral hemispheres and cerebral hemispheres. in the area of the temporal field (planum temporale), dysfunction in transnational cooperation, deficient development of directional function or even the incorrectly produced amount of neurotransmitters or malfunction in their use (Renteria, 2012).

The multifactorial approach, or cognitive perspective or psychological pedagogical model of interpretation of the phenomenon that is based on the multifactorial, is registered in the field of cognitive psychology, which studies every form of human behavior that includes the element of internal representation and information processing.

Based on this, the relevant theoretical positions and researches mainly concern issues such as: perceptual and motor deficits (visual-auditory perception, sensory integration, eye movement), memory, verbalphonological processing, deficits in the syntactic, semantic and lexical function of language, observance correct sequence or series of written symbols (serialization) etc. This separation into a single-factor and a multifactorial approach is a common practice for classifying related research in the international literature. Each of these interpretive approaches consists of a set of hypotheses that concern individual aspects of the whole problem (Renteria, 2012).

\section{Theories}


It is a fact that the group of disorders that has been researched the most from all special developmental learning difficulties, is that of dyslexic children. This is due to the increased incidence of the disorder but also to its centralized dimension, since it occurs primarily or secondarily with symptoms of hyperactivity, perceptual dysfunction and developmental insufficiency. This is why the term "dyslexia" is often used interchangeably with the term "learning disabilities". For these reasons, as well as the increased difficulty of finding bibliographic data for other specific developmental learning difficulties, more emphasis is placed on the causal theories of dyslexia. A fairly common view that prevails and is considered responsible for reading difficulties and dyslexia is that of cerebral dominance and pleurisy, and in particular the lack of dominance with a corresponding pleurisy picture. The term "ribbing" refers to the fact that the two sides of the brain perform different functions. The term "cerebral domination" refers to the correlation between the two hemispheres of the human brain and that one hemisphere dominates the other in terms of controlling the movements and functions of the body. In 1928, Samuel Orton proposed the theory of cerebral domination deficit or delayed tilt. The absence of cerebral domination simply means a lack of specialization and corresponding function of the left hemisphere of the brain in the linguistic behavior of the individual. In the case of the child with dyslexia, it is certainly not a question of the complete absence of such a characteristic but of a less clear dominance that has its impact on its linguistic function. The relevant deficit is part of the wider area of hemispherical differences and at the same time emphasizes the role of asymmetry of the brain in the developmental difficulties of the individual with particular reference to the use and understanding of language.

He himself hypothesized that there was a correlation between reading difficulty and a lack of cerebral dominance, and this led him to conclude:

(a) that readers with reading difficulties tend to reverse and mirror the mental image of sensory information in the brain;

(b) that the observed inversions and mirrors are associated with the symptom of lack of cerebral dominance, and

c) that this lack of brain dominance refers to differences in the organization of the brain of children with dyslexia, which fatally differentiates them from normal readers. Bakker (1990), who investigated the asymmetry of the hemispheres of the brain in relation to the cognitive process and especially to the reading process, found that at younger ages there was high-frequency activity in the right hemisphere, while at the age of about 7-8 years this activity was transferred to the left hemisphere. This phenomenon seemed to be related to reading performance. He then examined whether some children's failure to read was due to a delayed or no transfer of cognitive activity to the reading hemisphere after early childhood. He therefore assumed that some children do not, at the appropriate age, switch to the use of the left hemisphere, in which case there is a failure in the side of the lingual function in the predominant hemisphere or a reversal of the cerebral asymmetry. The same hypothesis is supported by Hier et. al., (1978) in their studies, where 10 out of 24 cases of "dyslexia" were found with reversal of cerebral asymmetry. This means that tilting leads to a hemisphere that is less properly prepared to support language functions, which is a risk factor for developing reading difficulties (Lagarde, et. al., 2009).

Based on empirical data, Witelson (1977) cites evidence that there are differences in the specialization of hemispheres between people with dyslexia and those without dyslexia in terms of their function in space. In particular, he argues that evolutionary dyslexia may be associated with a bilateral representation of the ability to perceive space and with a unambiguous representation of language functions in the left hemisphere. He concluded that the representation of the functions in the space that takes place in both hemispheres may disrupt the linguistic functions of the left hemisphere during the reading process. In other words, it is an interference of the right hemisphere in the functional role of the left hemisphere of the brain. That is, the organization of the brain in the dyslexic child is such that the right hemisphere is favored at the expense of the left. Something similar is supported by Denckla, Rudel \& Broman (1980), according to whom the structure of the brain organization is a transaction structure of a disadvantaged left with an advantageous right hemisphere.

Witelson (1987) proposed an almost purely neurobiological approach to the child's tongue. He argued that the right hemisphere of the child with dyslexia is somewhat deficient in the sense that his programmatic specialization in non-linguistic subjects is not observed. For this reason, the left hemisphere undertakes to make up for the familiar deficit of the right. As a result, the left hemisphere has a deficit in an area that is programmatically responsible. Thus, the intrusion of the left into the role of the right hemisphere simply means that the execution of the tasks of the right is undertaken by both hemispheres, which is why people with dyslexia tend to show mixed dominance of the hemispheres of the brain. Mixed dominance occurs in cases where a hemispheric dominance of one hemisphere has not been achieved, and this results in the creation of a symmetry, which is considered responsible for the onset of dyslexia. Many cases of mixed dominance and left-handedness or left-foot preference have been found even among non-dyslexic individuals. Critchley (1964), in a related study, argues that mixed ribs, without clear traces of brain dominance, can be considered as a more important 
causative factor of dyslexia. This hypothesis is supported by several researchers, including Cohen and Breolin (1984), Landwehrmeyer et al. (1990) and Brunswick and Rippon (1994), where in their study through provocative potential for examining transnational differences between normal and problem readers, reported results of greater symmetry in problem readers to a greater extent than in normal readers. This greater symmetry may represent a lower degree of hemispheric specialization in dyslexics resulting in reduced or delayed left hemisphere specialization in the processing of language stimuli (Baillieux, et. al., 2008).

Dyslexia, according to Critchley, must be linked to immaturity in brain development. The same is claimed by Zangwill (1962), but also by Satz and his colleagues (1987), where, according to them, a slow developmental maturation can suspend the dominance of the left hemisphere. In other words, areas of the brain of children with special learning-reading difficulties have not yet undergone the expected developmental changes, resulting in a slowdown in one or more learning areas. Delayed maturation of the left hemisphere is associated with developmental delay in the individual's perceptual, motor and language skills, related to reading and (spelling). Reading difficulties have indeed been associated, by some, with the delay in the maturation of the Central Nervous System, a hypothesis found in the late 19th century. This delay may be due to hereditary or acquired causes. However, the aforementioned authors believe that the observed developmental problems are related to "abnormalities" only in the growth rate, and there are no specific structural deficits in the Central Nervous System of the child. They essentially claim that when there is no cerebral asymmetry it does not always mean that there is a defect in the general structure of the brain.

Hemispherical asymmetries are a fundamental principle in the functional organization of the human brain. As has already been said, a neurologically normal brain exhibits the right hemisphere's superiority mainly in visual-spatial abilities, and the left hemisphere's specialization in language and motor skills. Deviations from this normal asymmetry indicate pathological changes within the two hemispheres and in the interaction between them. The transmission and consolidation of information between the cerebral hemispheres is thought to take place mainly through the midbrain, and the processes involved in reading and attention depend on the integrity of this interaction (Waldie \& Hausmannb, 2010). These two factors - the abnormal asymmetry of the hemispheres and the lesions in the mediastinum - have been linked after many studies to the etiology of developmental dyslexia. Njiokiktjien and colleagues (1994), in their study, speculate that the size of the medulla oblongata is associated with transnational cooperation and may contribute to the pathophysiological mechanisms that lead to learning difficulties. The midlobe is vulnerable during its development, as its shape and thickness are determined by genital and perinatal factors and thus its size is extremely variable. For example, children with dyslexia who have undergone a perinatal complication have a lower medulla oblongata, indicating a medullary lesion (Njiokiktjien, De Sonneville, \& Vaal, 1994). With regard to atypical cerebral asymmetry / morphology now, evidence has shown that developmental dyslexia is associated with it. Innovative research by Galaburda (1985) and his colleagues has revealed significant abnormalities that affect the left temporal-parietal region, such as neuronal ectopias, architectural (cellular) malformations, and a cerebral symmetry in the temporal temporal area (planum tempora). Hernandez, 2013).

These abnormalities were found in the brains of dying dyslexics, which appeared during the formation of the cerebral cortex, between the 5th and 6th month of pregnancy. Cortical cells have the ability to change orientation. Those who receive the appropriate stimuli fully develop, while those who do not receive the appropriate experience during the period of initial synaptogenesis, lose their specific orientation. The researchers therefore hypothesized that during this time the brain cells do not migrate to the extremities of the cortex and thus create abnormalities and malformations, mainly in the left hemisphere, where the language center is known. These "micro-dynasties", therefore, concentrated in this area, significantly disrupt the normal "architectural" shape of the dyslexic brain and remove the asymmetry commonly observed in the swollen lingual regions of the left temporal lobe and the smaller right hemisphere. Although such concentrations are also found in the brain of normal readers, they are rare and occur in the right anterior temporal cortex. This observed symmetry, therefore, is what has been blamed by many researchers for the impaired language development of children with dyslexia (Lagarde, et. al., 2009). The temporal field (planum temporale) is a triangular area of the cortex, located on the upper surface of the temporal lobe, next to the slit of the Silvius. It borders anteriorly to the Heschl helix and posteriorly to the end of the horizontal aspect of the Silvius slit. The temporal lobe is a large structure within the Wernicke area, known for its important role in language comprehension. It is also associated with the sloping of the tongue, due to the fact that it is one of the most sloping structures of the brain and usually has a sloping to the left in the brains of normal readers (SanchezBloom, 2013). Recent brain studies of children with developmental dyslexia have shown reduced asymmetry in the area of the temporal field in the left hemisphere, as the corresponding area of the right hemisphere appeared to be larger. This has led researchers to conclude that normal temporal field asymmetry is 
distorted in the brains of dyslexic children, which may lead to this deficit (Beaton, 1997; SanchezBloom, 2013). However, apart from the correlation of anatomical brain asymmetry with dyslexia, further research has focused on the functional asymmetry of the hemispheres. These studies revealed a reduced activity in the left temporal lobe in children and adults with developmental dyslexia during word recognition and phonological expression exercises. This dysfunction of activity in this area is accompanied by an inverted left-right asymmetry. In addition, it has been found that dyslexics with phonological processing problems are more likely to have cerebral symmetry, for example, in the temple area. The result, then, was to link the atypical size of the right temporal field in the brain of dyslexic individuals with their reduced phonological and speech abilities (Hernandez, 2013; Paul, 2005).

The reference to the lack of verbal skills is based on the acceptance that reading difficulties are focused on the verbal level. After all, language is a regulatory system of communication of man with his social environment that moves on a verbal level, that is, on a level of symbolic and mental representation. The fundamental component of language is symbolic in the sense that it enables it to transform sensory information into units of mental representation, which are then coded into appropriate verbal behavior and reaction. Reading can be considered to be composed of two skills: word recognition and content comprehension. For an effective reading the child must be able to recognize individual words of a text and to incorporate the meaning of the words and sentences for the comprehension of the reading text. Reading difficulties, therefore, can arise either from deficits in word decoding and content comprehension or from a combination of these two deficits. Theories linking dyslexia to vocabulary processing difficulties have been enthusiastically supported by scholars of the phenomenon, and their findings consistently establish that the child's predominant verbal deficits relate to the processes of verbal memory and phonemic division of syllables and words.

In order to acquire the reading ability and consequently the writing, it is necessary for the child to achieve the correspondence between the visual and the auditory representation of the words. This correspondence is achieved gradually through the process of graph-phoneme coupling, and is called phonological processing. The better the phonological processing, the better the reading and graphic performance. Phonological processing requires the development of at least three different skills:
a) phonological awareness,
b) phonological recoding and
c) voice recoding.

Of these three, the deficits in phonological awareness have been argued to coexist with the deficits of dyslexic children in reading but also that the relationship between them is causal (Ball, 1993; Lagarde, et. al., 2009).

According to phonological awareness deficit theory, a deficit in a lowerorder (phonological) language function prevents the transition to higherorder processes and the ability to draw meaning from the text. The problem with this is that the "poor" reader cannot use his highest language skills to make sense until the written word is first decoded and recognized. In other words, there is a problem first in decoding a word or a text and then in recognizing them (Kovelman, 2011; Shaywitz, 2001).

Although phonological awareness is more often recognized as a major deficit of dyslexia, findings from various studies have identified other types of processing deficits in this population, which calls into question the fact that dyslexia is due to an exclusive deficit in phonological processing. the heterogeneity of the subtypes of this population. A more well-known example is the deficit in rapid (automated) naming that often occurs in people with dyslexia and has been linked to reading. Naming speed is measured with a simple test, recording the time the person names objects, letters, etc. (Cronin, 2011, Park \& Lombardino, 2013, Savage, Pillay, \& Melidona, 2008). Based on this, in 1999, Wolf \& Bowers proposed the double deficit theory. This theory assumes that reading difficulty may be caused by deficits in phonological awareness, naming speed, or both. Explain why these two cognitive processes may be related is the fact that naming speed may reflect the ability to store or create spelling patterns, or that it effectively matches visual / spelling information with phonological codes or retrieves these codes quickly. from memory (Tiu, 2004; Vaessen \& Blomert, 2009).

However, in addition to the asymmetry of the temporal field and the damage to the parts of the cerebral cortex responsible for phonological processing mentioned above, research has revealed another area of the brain that may be responsible for the onset of learning disabilities. Traditionally, cerebellar function has been associated with movement control and coordination, but recently it has emerged as an important role in higher cognitive functions such as attention, memory, learning, executive control, language and visual spatial functions. Schmahmann (1996) was a major contributor to this finding, describing the involvement of the cerebellum in cognitive processing and its connection to the hemispheric and cortical points that aid in cognitive processing, as well as the connection of the cerebellar worm to structures. of the parietal system including the hippocampus and amygdala (Baillieux, 2008; Lagarde, 2009; Misciagna, 2012; Stoodley \& Stein, 2009; Vicari, 2012). Based on the above findings, neuroimaging studies have highlighted a possible involvement of the cerebellum with dyslexia. Indeed, in many 
studies of dyslexic individuals, a cerebellar symmetry and a high concentration of gray matter have been observed, suggesting cerebellar damage. Cerebellar symmetry has been linked by other studies to the severity of phonological difficulties and this was the reason for Nicolson and his colleagues (2001) to develop the theory of cerebellar deficit. According to her, automation by learned skills, such as articulation, reading, spelling, and phonological skills, is disrupted by a malfunction in the cerebellum (Baillieux, et. al., 2008; Stoodley \& Stein, 2012). In addition, cerebellar atrophy has been linked to writing deficits in children with dysgraphia. The writing ability includes two components, the linguistic and the kinetic. The first produces spelled words and the second converts abstract graphic information into kinetic instructions to execute graphic movements. Dysgraphia, then, is caused by a malfunction in one of these components, while a neurological disorder is blamed for it. Cerebellar damage is one of the hypotheses (Fournier del Castillo, et. al., 2010). The cerebellum has been blamed from time to time for other symptoms of dyslexia, such as disturbances in perception (audiovisual), short-term memory, serialization, ocular movement and balance. Initially, children's deficits in mental functions and activities such as visual perception and especially that concerning space, orientation and representation of shapes, copying complex designs, visual equation and visual discrimination of information, etc. compose the outline of the early hypotheses that substantiate the causal entity of dyslexia (Stoodley \& Stein, 2012). Satz (1978), referring to the ability of visual discrimination contained in his theory of reading difficulties, argue that perceptual-motor deficits are found in dyslexic children aged 5-8 years. In other words, researchers claim that children's visual perception difficulties are related to their chronological age. They also indicate that visual recognition and discrimination skills are the highest predictors of a child's reading performance. Deficiency in visual perception and visual memory is the most popular interpretation of dyslexia.

It was first given by Morgan (1896) and Hinshelwood (1900) and then promoted by Orton (1925). The latter paid special attention to the problems of orientation and sequence that are observed in the process of letter and word recognition, while disorders of this kind, he considered, to be a manifestation of slowed neurological development in the cerebral rib. Also, another possible cause is that associated with visual perceptual dysfunction regarding space and especially with the perception of shapes and images (Lagarde, et. al., 2009).

On the other hand, the observed deficits of children with dyslexia in auditory perception are also interesting. These deficits are not problems related to their acoustic acuity, but are involved with the stage of acoustic discrimination and acoustic coding. Studies show that reading problems stem from difficulties in processing and representing certain auditory abilities, which degrades the brain's ability to accurately participate in vital elements in the flow of speech. These difficulties impair a child's ability to match the sounds of speech with letters, a skill that is essentially particularly important for learning to read new words (Johnson, et. al., 2012).

Continuing the research, researchers of the problem of dyslexia investigated the possibility of a deficiency in the eye movement control systems of these children. Eye movement is a sensory indicator of a person's reading function. Javal (1879) was the first to report that in general readers do not observe a smooth flow of their eyes from left to right following the homonymous procedure and along the lines of the text. On the contrary, the reader's eyes move by making, in a sequential way, fast and abrupt jumps, making pauses at the same time, that is, focusing on certain parts of the text (Bellocchi, et. al., 2012).

Most studies today agree on the link between visual acuity and ocular motor control during reading. Attention seems to affect saccadic programming, that is, the position where the eyes will land on a word. In addition, these visual processes associated with attention are strictly related to normal and deficient reading. At the biological level, these deficits are attributed to dysfunction of the large cell pathway, which is supposed to be involved in low-spatial-frequency processing and ocular control. In general, research data that have studied the visual systems involved in reading with particular reference to the possibility that the combined movements of the individual's eyes, their incorrect concentration and various motor deficits create difficulties in reading, showed that deficient readers at slow or delayed reading make eye movements that are smaller in size compared to normal readers, last longer and form a schematic representation similar to that of the normal reader (Bellocchi, et. al., 2012)

Another issue that has preoccupied researchers with the etiology of dyslexia has to do with serialization and sequencing. Written language contains the element of order or sequence, and is therefore sequential, because its semantic rendering presupposes that its constituent elements, that is, letters and words, are arranged in a predetermined order. According to Thomson (1977), written language is composed of a series of "arbitrary symbols" that must be combined in the correct order in order to produce existing and familiar words in the spoken language. The correct order of the components of the written language is necessary, because, otherwise, there may be a complete change of its content.

Clinical observations and systematic descriptions of children with dyslexia confirm that these individuals have difficulty trying to serialize or control and monitor the observance of certain elements or symbols 
during the processing and presentation of information. These difficulties usually relate to cases that are intertwined with time and space or size, such as the observance of a particular series of letters and the sequential presentation of visual and audio material or, as mentioned, the observance of the exact sequence in which progressively the days of the week, months or seasons of the year, etc. The difficulties of people with dyslexia, according to Bakker (1967) stem from their residual perception of the time and order in which events occur. This theory even extends to countless issues. It is generally accepted that memory ability is a fundamental component of reading and that the development of shortterm auditory memory is associated with the development of reading ability. Research in recent years has linked working memory and shortterm memory processes to children's reading difficulties. According to theories that speak of perceptual deficit in people with dyslexia, children with low reading performance have significant deficits in visual memory and especially in the early stages of sensory processing of information received (Vaessen, et. al., 2009)

Vellutino (1979), based on theories about deficits in the child's visual memory, distinguishes two groups of people with low reading performance. The first group presents a basic memory impairment that is related to the initial stage of sensory processing of received information. The second group of readers with related difficulties presents a basic dysfunction that focuses on short-term memory and especially at the level of limited memory capacity but also deficit in decoding information and their internal verbal repetition. Similar research findings indicate that the performance of children with dyslexia in the use of short-term memory is lower than that of the normal reader (Lagarde, et. Al., 2009).

For the neurological review of the etiological theories of learning disabilities it is worth noting one more hypothesis. The angular helix of the brain is the protrusions between the grooves of the cerebral cortex whose function is to transmit and correlate the information recorded by visual stimuli with the stored / recorded phonemes-sounds of the tongue. It is, in other words, the area in the left hemisphere of the brain that is responsible for the written form of speech. It has been found that possible damage to the angular helix causes problems in reading and writing

\section{References}

1. Baillieux, H., Vandervliet, E. J., Manto, M., Parizel, P. M., De Deyn, P. P., \& Mariën, P. (2008). Developmental dyslexia and widespread activation across the cerebellar hemispheres. Brain \& Language (108).

2. Ball, E. W. (1993). Phonological awareness - What's important and to whom? Reading and Writing: An Interdisciplinary Journal (5).

3. Beaton, A. A. (1997). The Relation of Planum Temporale Asymmetry and Morphology of the Corpus Callosum to without at the same time affecting the person's speech. When a child has no learning difficulties, reads, the brain automatically correlates the symbols, which it sees on the book page, with the corresponding phonemes and puts them in the correct order to form the word. The brain of the child with special learning difficulties can not do this process properly and tries to read using other brain areas that serve the production of speech or its semantic processing. Therefore, in children with dyslexia, the Wernicke and angular helix areas - which are responsible for coding and decoding language and for writing, reading and understanding language - are malfunctioning or malfunctioning. While, on the contrary, the Broca area, the center of speech production, can be overworked.

\section{Conclusions}

As understood, based on the literature review, learning disabilities are a learning problem and learning is the result of a cognitive process and consequently of the coordinated function of the central nervous system. For this reason, the neurological basis of learning disabilities is fully understood. Starting from a lesion at a nodal point in the brain, an obstruction to the normal function of this area can be created, which may be the dominant area of some cognitive function that contributes to learning. Result is a problem in the learning process, and therefore in its expression. The problem of learning disabilities must be examined comprehensively, in order to have a complete picture and to be able to make a correct and timely intervention. Most research on the etiology of special learning disabilities revolves around a neurophysiological basis. Many of the theories developed around the various problems of children with learning disabilities have focused on specific areas of the brain that may be malfunctioning to substantiate their hypothesis. However, many times the brain findings are not localized and no specific damage can be detected focusing on an area of the brain. The difficulty of locating obvious brain damage in individuals who have been described as dyslexic has led to the strengthening of the hypothesis of the existence of a slight or minimal brain damage that cannot be easily diagnosed. This view has been modified by the hypothesis of mild or minimal brain dysfunction and reinforces the hypothesis of an undetectable brain injury, with the intention of covering heterogeneous groups of learning disabilities.

Handedness, Gender, and Dyslexia: A Review of the Evidence. BRAIN AND LANGUAGE (60).

4. Bellocchi, S., Muneaux, M., Bastien-Toniazzo, M., \& Ducrot, S. (2012). I can read it in your eyes: What eye movements tell us about visuo-attentional processes in developmental dyslexia. Research in Developmental Disabilities (34).

5. Cronin, V. S. (2011). RAN and Double-Deficit Theory. Journal of Learning Disabilities 46 (2).

6. Fournier del Castillo, M. C., Maldonado Belmonte, M. J., Ruiz-Falcó Rojas, M. L., López Pino, M. Á., Bernabeu 
Verdú, J., \& Suárez Rodríguez, J. M. (2010). Cerebellum Atrophy and Development of a Peripheral Dysgraphia: A Paediatric Case. Cerebellum (9).

7. Hernandez, N., Andersson, F., Edjlali, M., Hommet, C.,

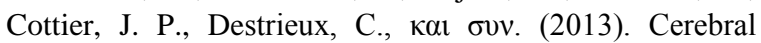
functional asymmetry and phonological performance in dyslexic adults. Psychophysiology (50).

8. Johnson, B. W., McArthur, G., Hautus, M., Reid, M., Brock, J., Castles, A., אal ovv. (2012). Lateralized auditory brain function in children with normal reading ability and in children with dyslexia. Neuropsychologia (51).

9. Kovelman, I., Norton, E. S., Christodoulou, J. A., Gaab, N.,

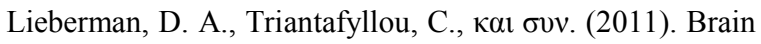
Basis of Phonological Awareness for Spoken Language in Children and Its Disruption in Dyslexia. Cerebral Cortex (22).

10. Lagarde, J., Hantkie, O., Hajjioui, A., \& Yelnik, A. (2009). Neuropsychological disorders induced by cerebellar damage. Annals of Physical and Rehabilitation Medicine (52).

11. Misciagna, S. (2012). Cerebellar contribution to cognitive, emotional, and behavioural functions in children with cerebellar abnormalities. Developmental Medicine \& Child Neurology (53).

12. Njiokiktjien C., De Sonneville, L., \& Vaal, J. (1994). Callosal size in children with learning disabilities. Behavioural Brain Research (64).

13. Park, H., \& Lombardino, L. J. (2013). Relationships among cognitive deficits and component skills of reading in younger and older students with developmental dyslexia. Research in Developmental Disabilities (34).

14. Paul, I., Bott, C., Heim, S., Eulitz, C., \& Elbert, T. (2005). Reduced hemispheric asymmetry of the auditory $\mathrm{N} 260 \mathrm{~m}$ in dyslexia. Neuropsychologia (44).
15. Renterı, M. E. (2012). Cerebral Asymmetry: A Quantitative, Multifactorial, and Plastic Brain Phenotype. Twin Research and Human Genetics (15).

16. SanchezBloom, J., Garcia-Barrera, M. A., Miller, C. J., Miller, S. R., \& Hynd, G. W. (2013). Planum temporale morphology in children with developmental dyslexia. Neuropsychologia (51).

17. Savage, R., Pillay, V., \& Melidona, S. (2008). Rapid Serial Naming Is a Unique Predictor of Spelling in Children. Journal of Learning Disabilities (41).

18. Shaywitz, B. A., Shaywitz, S. E., Pugh, K. R., Fulbright, R. K., Mencl, W. E., Constable, R. T., kal ovv. (2001). The neurobiology of dyslexia. Clinical Neuroscience Research (1).

19. Stoodley, C. J., \& Stein, J. F. (2012). Cerebellar Function in Developmental Dyslexia. Cerebellum (12).

20. Stoodley, C. J., \& Stein, J. F. (2009). The cerebellum and dyslexia. Cortex (47).

21. Tiu, R. D., Wadsworth, S. J., Olson, R. K., \& DeFries, J. C. (2004). Causal Models of Reading Disability: A Twin Study. Twin Research (7).

22. Vaessen, A., \& Blomert, L. (2009). Long-term cognitive dynamics of fluent reading development. Journal of Experimental Child Psychology (105).

23. Vicari, S., Marotta, L., Menghini, D., Molinari, M., \& Petrosini, L. (2012). Implicit learning deficit in children with developmental dyslexia. Neuropsychologia (41).

24. Waldie, K. E., \& Hausmannb, M. (2010). Right frontoparietal dysfunction in children with ADHD and developmental dyslexia as determined by line bisection judgements. Neuropsychologia (48).
This work is licensed under Creative Commons Attribution 4.0 License

To Submit Your Article Click Here: Submit Manuscript

DOI:10.31579/2578-8868/165
Ready to submit your research? Choose Auctores and benefit from:

* fast, convenient online submission

* rigorous peer review by experienced research in your field

* rapid publication on acceptance

* authors retain copyrights

* unique DOI for all articles

* immediate, unrestricted online access

At Auctores, research is always in progress.

Learn more www.auctoresonline.org/journals/neuroscience-andneurological-surgery 